Bo Wang, Yu Zhou, Yu Wang, Gao-Hui Chen, Qi-Xin Lian and Hai-Dong Wang*

\title{
Crystal structure of poly[( $\mu_{2}-1,3-$ bis(imidazol- 1-ylmethyl)benzene- $\left.\mathrm{K}^{2} \mathrm{~N}: \mathrm{N}^{\prime}\right)\left(\right.$ nitrato- $\mathrm{K}^{1} O$ ) cadmium(II)] - water (2/1), $\mathrm{C}_{28} \mathrm{H}_{32} \mathrm{CdN}_{10} \mathrm{O}_{7}$
}

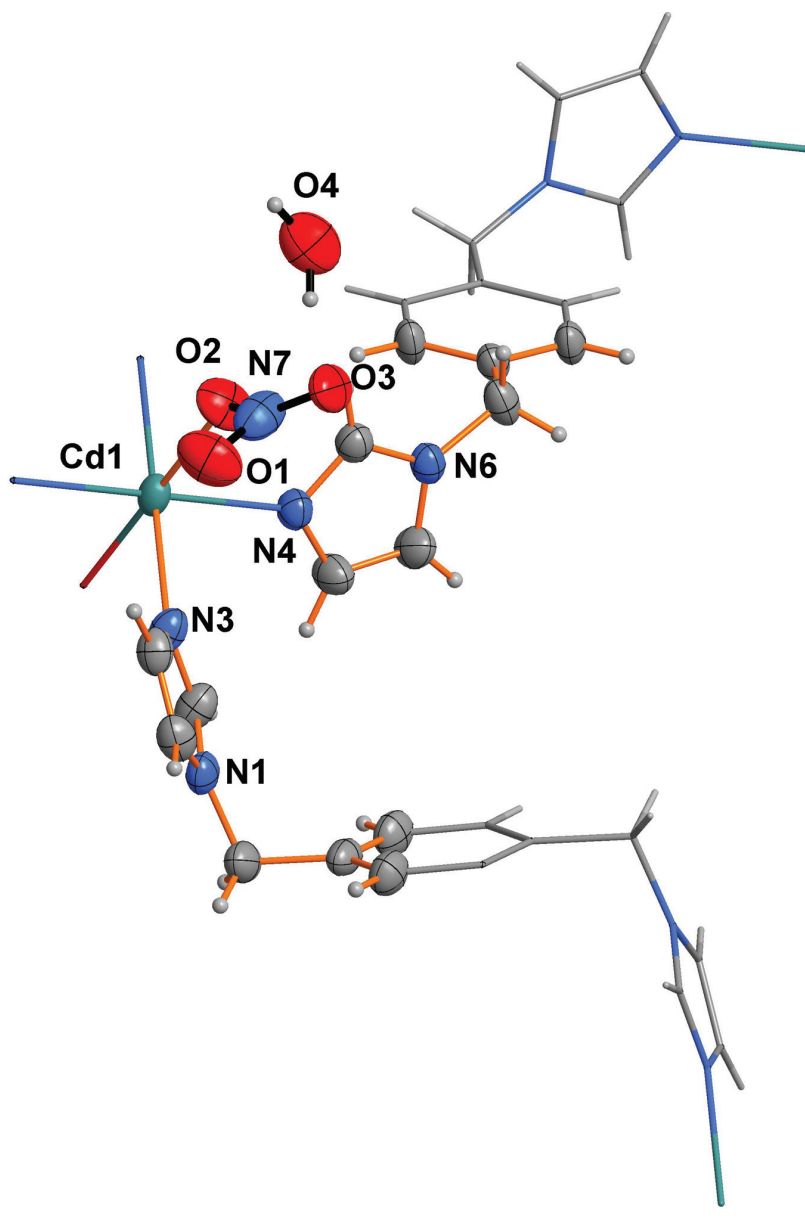

https://doi.org/10.1515/ncrs-2019-0126

Received February 20, 2019; accepted April 5, 2019; available online April 27, 2019

\footnotetext{
*Corresponding author: Hai-Dong Wang, Department of General Surgery, Daqing People's Hospital, Daqing, Heilongjiang, China, e-mail: haidong_wang66@126.com

Bo Wang, Yu Zhou and Qi-Xin Lian: Department of Oncology, First Affiliated Hospital of Jiamusi University, Jiamusi, Heilongjiang, China

Yu Wang: Department of Anorectal Surgery, First Affiliated Hospital of Jiamusi University, Jiamusi, Heilongjiang, China Gao-Hui Chen: Jiamusi University, Jiamusi, Heilongjiang, China
}

\begin{abstract}
$\mathrm{C}_{28} \mathrm{H}_{32} \mathrm{CdN}_{10} \mathrm{O}_{7}$, triclinic, $P \overline{1} \quad$ (no. 2), $\quad a=8.2649$ (5) $\AA$, $b=9.7841(6) \AA, \quad c=10.0808(6) \AA, \quad \alpha=94.069(7)^{\circ}$, $\beta=104.912(7)^{\circ}, \quad \gamma=97.695(7)^{\circ}, \quad V=775.89(9) \AA^{3}, \quad Z=1$, $R_{\mathrm{gt}}(F)=0.0432, w R_{\text {ref }}\left(F^{2}\right)=0.1140, T=293(2) \mathrm{K}$.
\end{abstract}

CCDC no.: 1908062

A part of the title structure is shown in the figure. Table 1 contains crystallographic data and Table 2 contains the list of the atoms including atomic coordinates and displacement parameters.

Table 1: Data collection and handling.

\begin{tabular}{ll}
\hline Crystal: & Colourless block \\
Size: & $0.41 \times 0.23 \times 0.12 \mathrm{~mm}$ \\
Wavelength: & Mo $K \alpha$ radiation $(0.71073 \AA$ A $)$ \\
$\mu:$ & $0.77 \mathrm{~mm}^{-1}$ \\
Diffractometer, scan mode: & Xcalibur, $\omega$ \\
$\theta_{\text {max }}$, completeness: & $27.5^{\circ},>99 \%$ \\
$N(h k l)_{\text {measured }}, N(h k l)_{\text {unique }}, R_{\text {int }}:$ & $12375,3552,0.023$ \\
Criterion for $I_{\text {obs }}, N(h k l)_{\mathrm{gt}}:$ & $I_{\text {obs }}>2 \sigma\left(I_{\text {obs }}\right), 3382$ \\
$N(\text { param })_{\text {refined }}:$ & 242 \\
Programs: & Olex2 [1], SHELX [2], \\
& CrysAlis ${ }^{\text {PRO }[3]}$ \\
\hline
\end{tabular}

\section{Source of material}

A mixture of 1,3-bis(imidazol-1-ylmethyl)benzene $(3.0 \mathrm{mg}$ ), $\mathrm{Cd}\left(\mathrm{NO}_{3}\right)_{2} \cdot 4 \mathrm{H}_{2} \mathrm{O}(4.5 \mathrm{mg})$, and $5 \mathrm{~mL}$ mixed solution of $\mathrm{EtOH} / \mathrm{H}_{2} \mathrm{O}(v / v=1 / 4)$ was added to a hard glass tube, pumped to a near-vacuum, heated at $135{ }^{\circ} \mathrm{C}$ for $48 \mathrm{~h}$, and cooled to $25{ }^{\circ} \mathrm{C}$ with a decreasing rate of $3{ }^{\circ} \mathrm{C} / \mathrm{h}$. Colorless block crystals were obtained with the yield of ca. $42 \%$ (based on 1,3-bis(imidazol-1-ylmethyl)benzene).

\section{Experimental details}

$\mathrm{H}$ atoms were positioned geometrically and refined using a riding model. There is a disorder of the nitrato ligand (Table 2).

\section{Comment}

Coordination polymers have gained great interests due to their structural and applications in some fields such as

This work is licensed under the Creative Commons Attribution 4.0 Public License. 
Table 2: Fractional atomic coordinates and isotropic or equivalent isotropic displacement parameters $\left(\AA^{2}\right)$.

\begin{tabular}{|c|c|c|c|c|}
\hline Atom & $x$ & $y$ & $z$ & $U_{\text {iso }} * / U_{\text {eq }}$ \\
\hline Cd1 & 0.000000 & 0.000000 & 0.000000 & $0.04795(13)$ \\
\hline $01^{a}$ & $-0.2772(8)$ & $0.0498(8)$ & $0.2279(6)$ & $0.132(2)$ \\
\hline $01^{b}$ & $-0.3226(16)$ & $0.1908(14)$ & $0.0151(11)$ & $0.108(5)$ \\
\hline $\mathrm{H} 4 \mathrm{C}$ & -0.400643 & 0.182846 & -0.102156 & $0.130^{\star}$ \\
\hline H4D & -0.562983 & 0.107196 & -0.196696 & $0.130 *$ \\
\hline $02^{\mathrm{a}}$ & $-0.2504(6)$ & $0.0745(6)$ & $0.0318(5)$ & $0.1002(15)$ \\
\hline $02^{\mathrm{b}}$ & $-0.360(4)$ & $0.154(3)$ & $0.211(2)$ & $0.135(7)$ \\
\hline $03^{a}$ & $-0.3672(6)$ & $0.2199(5)$ & $0.1317(5)$ & $0.0897(14)$ \\
\hline $03^{b}$ & $-0.241(3)$ & $0.0196(15)$ & $0.098(2)$ & $0.096(4)$ \\
\hline $04^{c}$ & $-0.4762(14)$ & $0.1718(11)$ & $-0.1816(9)$ & $0.138(3)$ \\
\hline N1 & $0.3707(4)$ & $0.1259(3)$ & $0.4058(3)$ & $0.0538(6)$ \\
\hline N3 & $0.1566(4)$ & $0.0438(3)$ & $0.2270(3)$ & $0.0565(6)$ \\
\hline N4 & $0.0856(4)$ & $0.2240(3)$ & $-0.0479(3)$ & $0.0536(6)$ \\
\hline N6 & $0.0511(4)$ & $0.4201(3)$ & $-0.1366(3)$ & $0.0507(6)$ \\
\hline N7 & $-0.2960(5)$ & $0.1220(4)$ & $0.1235(4)$ & $0.0850(11)$ \\
\hline C1 & $-0.0108(5)$ & $0.3821(3)$ & $-0.4344(3)$ & $0.0567(8)$ \\
\hline H1 & -0.017826 & 0.301944 & -0.390476 & $0.068^{\star}$ \\
\hline C14 & $-0.0254(4)$ & $0.2989(3)$ & $-0.1127(3)$ & $0.0532(7)$ \\
\hline H14 & -0.142154 & 0.270627 & -0.138585 & $0.064^{\star}$ \\
\hline $\mathrm{C} 2$ & $0.1108(5)$ & $0.0191(3)$ & $0.3445(4)$ & $0.0562(7)$ \\
\hline $\mathrm{H} 2$ & 0.005654 & -0.026257 & 0.347579 & $0.067^{\star}$ \\
\hline C3 & $-0.0005(5)$ & $0.6264(3)$ & $-0.4312(3)$ & $0.0557(7)$ \\
\hline H3 & -0.000513 & 0.712281 & -0.385265 & $0.067^{\star}$ \\
\hline C4 & $-0.0311(5)$ & $0.5209(4)$ & $-0.2192(3)$ & $0.0614(8)$ \\
\hline $\mathrm{H} 4 \mathrm{~A}$ & 0.017762 & 0.613801 & -0.174699 & $0.074^{\star}$ \\
\hline $\mathrm{H} 4 \mathrm{~B}$ & -0.150796 & 0.506956 & -0.223130 & $0.074^{\star}$ \\
\hline C5 & $0.2387(5)$ & $0.3023(4)$ & $-0.0314(4)$ & $0.0652(9)$ \\
\hline H5 & 0.342062 & 0.276167 & 0.011061 & $0.078^{\star}$ \\
\hline C6 & $0.2208(5)$ & $0.4237(4)$ & $-0.0852(4)$ & $0.0653(9)$ \\
\hline $\mathrm{H} 6$ & 0.306675 & 0.494913 & -0.086801 & $0.078^{\star}$ \\
\hline $\mathrm{C} 7$ & $0.3155(5)$ & $0.1074(4)$ & $0.2699(4)$ & $0.0619(8)$ \\
\hline $\mathrm{H} 7$ & 0.380678 & 0.135750 & 0.211182 & $0.074^{\star}$ \\
\hline $\mathrm{C} 8$ & $0.2395(5)$ & $0.0697(4)$ & $0.4549(3)$ & $0.0597(8)$ \\
\hline $\mathrm{H} 8$ & 0.239989 & 0.067265 & 0.547091 & $0.072^{\star}$ \\
\hline C9 & $0.5332(5)$ & $0.2088(4)$ & $0.4847(4)$ & $0.0649(9)$ \\
\hline $\mathrm{H} 9 \mathrm{~A}$ & 0.620592 & 0.193329 & 0.439533 & $0.078^{*}$ \\
\hline H9B & 0.565688 & 0.179883 & 0.576666 & $0.078^{\star}$ \\
\hline C10 & $0.5186(4)$ & $0.3608(3)$ & $0.4949(4)$ & $0.0540(7)$ \\
\hline C11 & $0.4718(5)$ & $0.4228(4)$ & $0.6033(4)$ & $0.0635(8)$ \\
\hline H11 & 0.453137 & 0.371399 & 0.673567 & $0.076^{\star}$ \\
\hline C12 & $0.5476(5)$ & $0.4380(4)$ & $0.3921(4)$ & $0.0630(8)$ \\
\hline $\mathrm{H} 12$ & 0.580364 & 0.396922 & 0.319125 & $0.076^{\star}$ \\
\hline C13 & $-0.0119(4)$ & $0.5087(3)$ & $-0.3641(3)$ & $0.0480(6)$ \\
\hline
\end{tabular}

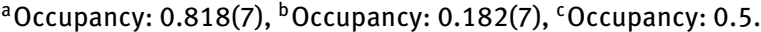

fluorescent probes and magnetism [4]. The suitable organic ligands were used in the preparing coordination polymer which had become an important way. Herein, we selected 1,3-bis(imidazol-1-ylmethyl)benzene to synthesize a Cd(II) coordination polymer.

The primary structural unit contains one half of a $\mathrm{Cd}^{2+}$ cation, one 1,3-bis(imidazol-1-ylmethyl)benzene ligands, one $\mathrm{NO}_{3}{ }^{-}$anions and one half occupied isolated water molecule. In the structure, the $\mathrm{Cd}^{2+}$ cation is six-coordinated by four nitrogen atoms from four different 1,3-bis(imidazol-1ylmethyl)benzene ligands and two oxygen atoms from two $\mathrm{NO}_{3}{ }^{-}$anions. By means of such connection modes, a $2 \mathrm{D}$ framework is formed. In the framework, each $\mathrm{Cd}^{2+}$ cation is surrounded by four 1,3-bis(imidazol-1-ylmethyl)benzene ligands and two $\mathrm{NO}_{3}{ }^{-}$anions. The features of the structure are similar with the known compounds $\left[\mathrm{Cd}\left(\mathrm{NO}_{3}\right)_{2}\left(\mathrm{C}_{14} \mathrm{H}_{14} \mathrm{~N}_{4}\right)_{2}\right]_{\mathrm{n}}$ [5], [ $\left.\mathrm{C}_{28} \mathrm{H}_{28} \mathrm{MnN}_{14}\right]_{n}$ [6] and $\left[\mathrm{C}_{33} \mathrm{H}_{32} \mathrm{MnN}_{14} \mathrm{O}\right]_{n}$ [6]. The bond lengths of $\mathrm{Cd}-\mathrm{N}$ and $\mathrm{Cd}-\mathrm{O}$ are all in their normal ranges and they can be compared with previously reported $\mathrm{Cd}(\mathrm{II})$ coordination polymers $[7,8]$.

\section{References}

1. Dolomanov, O. V.; Bourhis, L. J.; Gildea, R. J.; Howard, J. A. K.; Puschmann, H.: OLEX2: a complete structure solution, refinement and analysis program. J. Appl. Crystallogr. 42 (2009) 339-341.

2. Sheldrick, G. M.: Crystal structure refinement with SHELXL. Acta Crystallogr. C71 (2015) 3-8.

3. Agilent Technologies. CrysAlis PRO. Version 1.171.35.15. Agilent Technologies UK Ltd, Oxford, UK (2011).

4. Shanmugaraju, S.; Dabadie, C.; Byrne, K.; Savyasachi, A. J.; Umadevi, D.; Schmitt, W.; Kitchen, J. A.; Gunnlaugsson, T. A.: Supramolecular Troger's base derived coordination zinc polymer for fluorescent sensing of phenolicnitroaromatic explosives in water. Chem. Sci. 8 (2017) $1535-1546$.

5. Li, F.-A.; Yang, W.-C.; Hu, X.-M.: Crystal structure of bisnitrate-bis(1-(4-((1H-imidazol-1-yl)methyl)-benzyl)-1Himidazole)cadmium(II), [Cd( $\left.\left(\mathrm{NO}_{3}\right)_{2}\left(\mathrm{C}_{14} \mathrm{H}_{14} \mathrm{~N}_{4}\right)_{2}\right]_{\mathrm{n}}$. Z. Kristallogr. NCS 226 (2011) 587-588.

6. Gao, E-Q.; Xu, Y.-X.; Yan, C.-H.: Two square grid coordination polymers with manganese(II) and 1,4-bis(imidazole-1-ylmethyl) benzene. CrystEngComm. 6 (2004) 298-302.

7. Lin, Z.-J.; Lü, J.; Hong, M.; Cao, R.: Metal-organic frameworks based on flexible ligands (FL-MOFs): structures and applications. Chem. Soc. Rev. 43 (2014) 5867-5895.

8. Zhang, J.; Huo, L.; Wang, X.; Fang, K.; Fan, L.; Hu, T.: Structural diversity, magnetic properties, and luminescent sensing of the flexible tripodal ligand of 1,3,5-tris (4-carbonylphenyloxy)benzene based $\mathrm{Mn}(\mathrm{II}) / \mathrm{Cd}(\mathrm{II})$ coordination polymers. Cryst. Growth Des. 17 (2017) 5887-5897. 